\title{
Comparative effectiveness of immunosuppressive drugs and corticosteroids for lupus nephritis: a systematic review and network meta-analysis
}

\author{
Jasvinder A. Singh ${ }^{1,2,3^{*}}$, Alomgir Hossain ${ }^{4}$, Ahmed Kotb ${ }^{4}$ and George A. Wells ${ }^{4}$
}

\begin{abstract}
Background: There is a lack of high-quality meta-analyses and network meta-analyses of immunosuppressive drugs for lupus nephritis. Our objective was to assess the comparative benefits and harms of immunosuppressive drugs and corticosteroids in lupus nephritis.

Methods: We conducted a systematic review and network meta-analysis (NMA) of trials of immunosuppressive drugs and corticosteroids in patients with lupus nephritis. We calculated odds ratios (OR) and $95 \%$ credible intervals (Crl).

Results: Sixty-five studies that met inclusion and exclusion criteria; data were analyzed for renal remission/response (37 trials; 2697 patients), renal relapse/flare (13 studies; 1108 patients), amenorrhea/ovarian failure (eight trials; 839 patients) and cytopenia (16 trials; 2257 patients). Cyclophosphamide [CYC] low dose (LD) and CYC high-dose (HD) were less likely than mycophenolate mofetil [MMF] and azathioprine [AZA], CYC LD, CYC HD and plasmapharesis less likely than cyclosporine [CSA] to achieve renal remission/response. Tacrolimus [TAC] was more likely than CYC LD to achieve renal remission/response. MMF and CYC were associated with a lower odds of renal relapse/flare compared to PRED and MMF was associated with a lower rate of renal relapse/flare than AZA. CYC was more likely than MMF and PRED to be associated with amenorrhea/ovarian failure. Compared to MMF, CYC, AZA, CYC LD, and CYC HD were associated with a higher risk of cytopenia.

Conclusions: In this systematic review and NMA, we found important differences between immunosuppressives used for the treatment of lupus nephritis. Patients and physicians can use this information for detailed informed consent in a patient-centered approach. Study limitations of between-study clinical heterogeneity and small sample size with type II error must be considered when interpreting these findings.
\end{abstract}

Systematic review registration: PROSPERO: CRD42016032965

Keywords: Network meta-analysis, Immunosuppressive drugs, Glucocorticoids, Lupus nephritis, Lupus, Renal remission, Renal relapse, Tacrolimus, Cyclophosphamide, Mycophenolate mofetil

\footnotetext{
* Correspondence: Jasvinder.md@gmail.com

'Medicine Service, VA Medical Center, Birmingham, AL, USA

2Department of Medicine at the School of Medicine, and Division of

Epidemiology at the School of Public Health, University of Alabama at

Birmingham, Faculty Office Tower 805B, 510 20th Street S, Birmingham, AL

35294, USA

Full list of author information is available at the end of the article
} 


\section{Background}

Lupus is a chronic autoimmune disease that frequently involves the kidney. Lupus nephritis can lead to kidney failure, dialysis, and even premature death if not treated appropriately. Lupus primarily affects young women and is more common and more severe in racial/ethnic minorities, who experience worse outcomes [1-5].

Comparative effectiveness research (CER) of drugs used to treat lupus nephritis is an imperative [6]. Patients, facing difficult decisions related to their treatment options, such as those related to life- and/or organthreatening clinical situations (active lupus nephritis, for example) need information about possible harms and benefits of available treatment options in a format that provides comparisons of multiple treatment options.

The 2012 American College of Rheumatology (ACR) lupus nephritis treatment guidelines [7] and the Cochrane systematic review of interventions for lupus nephritis [8] assessed literature up to 2010 and 2012, respectively. However, indirect comparisons were not performed in either. Few lupus nephritis treatments have been compared directly in clinical trials. This leaves a large knowledge gap. Clinicians and patients have to choose between various immunosuppressive drugs in the absence of such knowledge. Therefore, we need evidence synthesis using valid methods to incorporate indirect and direct comparisons of efficacy/harms of these treatments.

A state-of-the-art network meta-analysis (NMA), with updated information, is a necessary precursor to the development of a clinical decision-making tool for physician and their patients with lupus nephritis. This information can be very helpful to patients during the treatment decision-making process for new disease, disease flare or refractory disease. It is not surprising that for a rare condition such as lupus with roughly 161,000 patients in the USA [9], most multicenter trials have $<500$ patients (often 50-200 patients). This makes many trials underpowered for assessing treatment-related differences in disease outcomes.

One useful approach is the use of composite outcomes, which have been widely used to address important clinical questions in obstetrics, cardiology, and other disciplines [10-12]. Assessments of treatment options using composite outcomes can help answer important question in a timely fashion without requiring studies with large sample sizes. This study aimed to perform a systematic review and NMA to compare benefits and harms of immunosuppressive drugs compared to each other and to corticosteroids focusing on four composite benefit/harm outcomes: (1) renal remission/response; (2) renal relapse/flare; (3) ovarian failure/amenorrhea; and (4) bone marrow toxicity.

\section{Methods}

We used rigorous methods for the systematic review and NMA based on the Agency for Healthcare Research and Quality (AHRQ) recommendations [13], the Cochrane handbook [14], and the PRISMA guidelines. The Institutional Review Board at the University of Alabama at Birmingham (UAB) approved the study. The need for informed consent was waived for this systematic review, since no human subjects were involved. The study protocol was registered in PROSPERO, CRD42016032965 (http://www.crd.york.ac.uk/PROSPERO/).

\section{Methods for systematic review: study eligibility criteria, outcomes and data abstraction}

This systematic review included randomized controlled trials (RCTs) or controlled clinical trials (CCTs) of immunosuppressive drugs or corticosteroids for lupus nephritis, published in English that reported any safety or efficacy outcome. Included medications were: corticosteroids [PRED]; cyclophosphamide [CYC]; mycophenolate mofetil [MMF]; azathioprine [AZA]; cyclosporine [CSA]; tacrolimus [TAC]; or rituximab [RTX], Belimumab studies could not be included in this systematic review since these studies included patients with lupus, and only a small proportion had active lupus nephritis. A Cochrane systematic review of belimumab for lupus in underway [15]. There were no restrictions with regards to the medication dose or the duration of medication use.

Experienced librarians (JJ and TR) updated two systematic reviews [7, 8] from their search end dates (August 2010 and April 2012, respectively) to September 2013 using the PubMed database. The search used the following terms: (Lupus[text word] OR "Lupus Vulgaris"[MeSH] OR "Lupus Erythematosus, Cutaneous"[MeSH] OR "Lupus Erythematosus, Systemic"[Mesh]) AND ("Kidney Diseases"[MeSH] OR nephropath*[text word] OR Transplants[MeSH] OR Transplantation[MesH] OR transplantation[subheading] OR transplant*[text word] OR "Kidney"[Mesh] OR Kidney*[text word] OR Renal"[text word] OR "End Stage Renal Disease"[text word] OR ESRD[text word] OR Glomerulonephr*[text word] OR "GN"[text word] OR "crescentic GN"[text word]) NOT ("animals"[MeSH] NOT "humans"[MeSH]).

Raw data abstracted for the ACR lupus nephritis guidelines systematic review [7] were obtained (courtesy Dr. Jennifer Grossman (JG), see acknowledgment section), or were abstracted from the Revman tables of the Cochrane Systematic Review [8]. A librarian $(\mathrm{CH})$ also performed a search for all lupus trials for harms (for conditions other than lupus nephritis) in PubMed and SCOPUS from inception to February 2014, based on an a priori assumption that treatmentrelated harms may not depend on whether kidney is involved or not. Examination of data from this search 
revealed little additive data for harms, but added clinical heterogeneity related to differences in patient population. Therefore, after careful consideration of pros and cons, we decided not to use these data in analyses.

The PICO (patient, intervention, comparator, outcome) for our systematic review and NMA were defined as follows:

P: Adults 18 years or older, meeting the 1987 American College of Rheumatology Classification criteria for systemic lupus erythematosus (SLE) [16], who have lupus nephritis.

I: Immunosuppressant drug alone or in combination with other immunosuppressant drugs or biologics (such as rituximab) or corticosteroids. Medication doses were categorized as low, standard or high dose/ duration (LD, SD and HD).

C: Placebo or another immunosuppressive with/ without biologic.

O: Benefit and harm outcomes (renal remission/ response, renal relapse/flare, fertility, bone marrow suppression), defined as follows.

Benefits were assessed by two composite outcomes (for detailed definitions of components, see Additional file 1): (1) renal remission/response (indicating success of therapy): included complete renal remission, [17] partial renal remission [18, 19] and renal response; (2) renal relapse/flare (indicating failure of therapy): included renal relapse [20] and renal flare (Additional file 1). Harms were assessed by two composite outcomes: (1) ovarian failure/ amenorrhea: included ovarian failure and amenorrhea; and (2) bone marrow toxicity: cytopenia including leucopenia.

Two trained abstractors (AO, $\mathrm{AB}$ ) independently reviewed abstracts and titles, abstracted data in duplicate directly into Microsoft excel sheets and assessed the risk of bias according to the Cochrane risk of bias tool [21]. We examined the following domains as low or high risk of bias or unclear risk (lack of information or uncertainty about potential for bias): randomization sequence generation, allocation sequence concealment, blinding of participants, personnel and outcome assessors, incomplete outcome data (primary outcome data reporting, dropout rates and reasons for withdrawal, appropriate imputation of missing data, an overall completion rate $\geq 80 \%$ ), and selective outcome reporting and other potential threats to validity (considering external validity, e.g., relevant use of co-interventions, bias due to funding source). An adjudicator (JS) resolved any disagreements not resolved by consensus. An expert rheumatologist (JS) and an expert in lupus (JG) examined for similarity of studies prior to performing evidence synthesis by the examination of similarity of study population and interventions.

We designated doses as follows: (1) CYC: standarddose/duration , SD: $0.5-1.0 \mathrm{gm} / \mathrm{m}^{2}$ intravenously (IV) q month for $6-12$ months or $2-2.5 \mathrm{mg} / \mathrm{kg}$ orally (PO) daily $\times$ 3-6 months; high-dose/duration, HD: dose higher or duration longer than SD; low-dose/duration, LD: lower dose or duration shorter than SD, including the EUROlupus dose, $500 \mathrm{mg}$ IV q14 days $\times 6$ doses (mean $3 \mathrm{~g}$ ); (2) AZA: SD, $1-3 \mathrm{mg} / \mathrm{kg}$ po daily; $\mathrm{HD},>3 \mathrm{mg} / \mathrm{kg}$ po daily; (3) LEF: $1 \mathrm{mg} / \mathrm{kg}$ po $\mathrm{qd} \times 3$ days then $30 \mathrm{mg}$

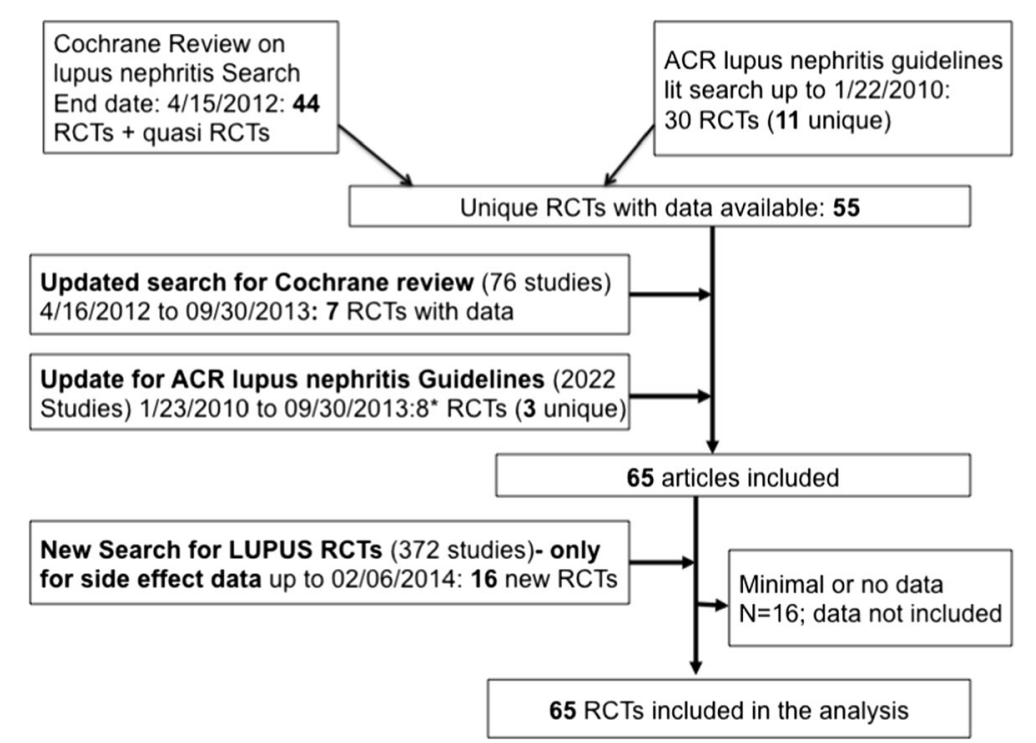

Fig. 1 PRISMA study flow chart for study selection. Legend: none 
po qd x 6 months; and (4) PRED: SD, prednisone/ methylprednisolone $1 \mathrm{gm} / \mathrm{m}^{2}$ IV q month $\times 6$ months or prednisone $60 \mathrm{mg}$ po qd 1-3 months then tapered over 3-12 months as tolerated; HD, prednisone/methylprednisolone $1 \mathrm{gm} / \mathrm{m}^{2}$ qd IV $\times 3$, then one dose $\mathrm{q}$ month for 1 year or prednisone $1 \mathrm{mg} / \mathrm{kg}$ daily for 4-
8 weeks (or unspecified period). When dose is not specified, medication dose is the standard dose.

\section{Bayesian network meta-analysis (NMA)}

We used Bayesian mixed treatment comparison (MTC) meta-analyses [22-24] to assess the comparative
Renal remission or renal response

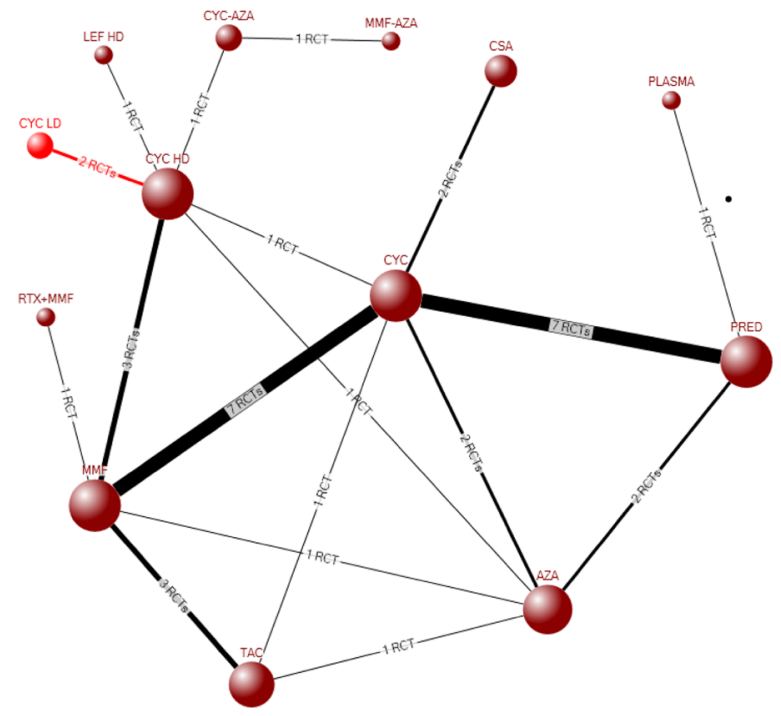

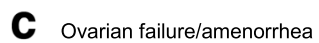

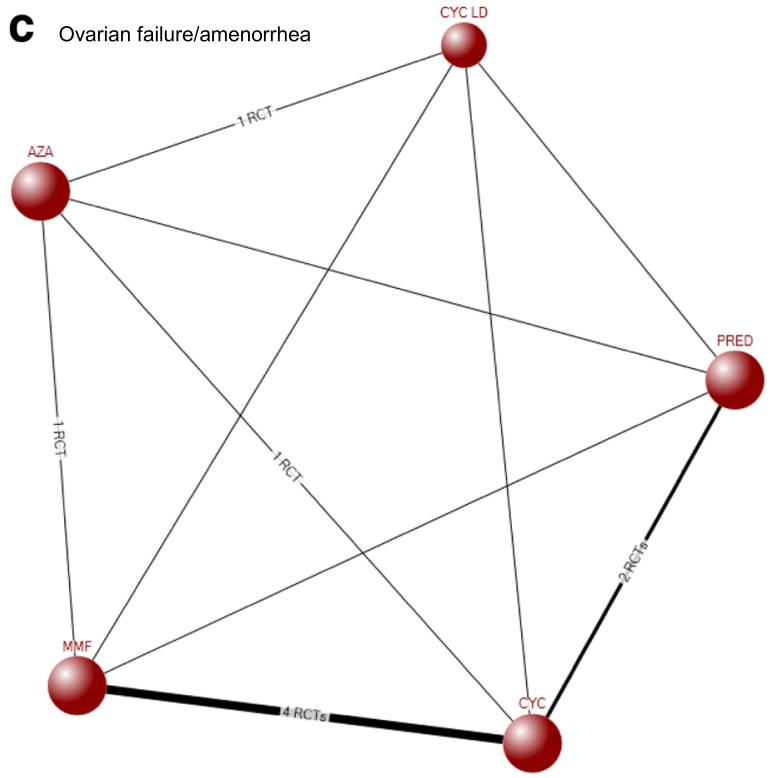

Denal relapse or renal flare

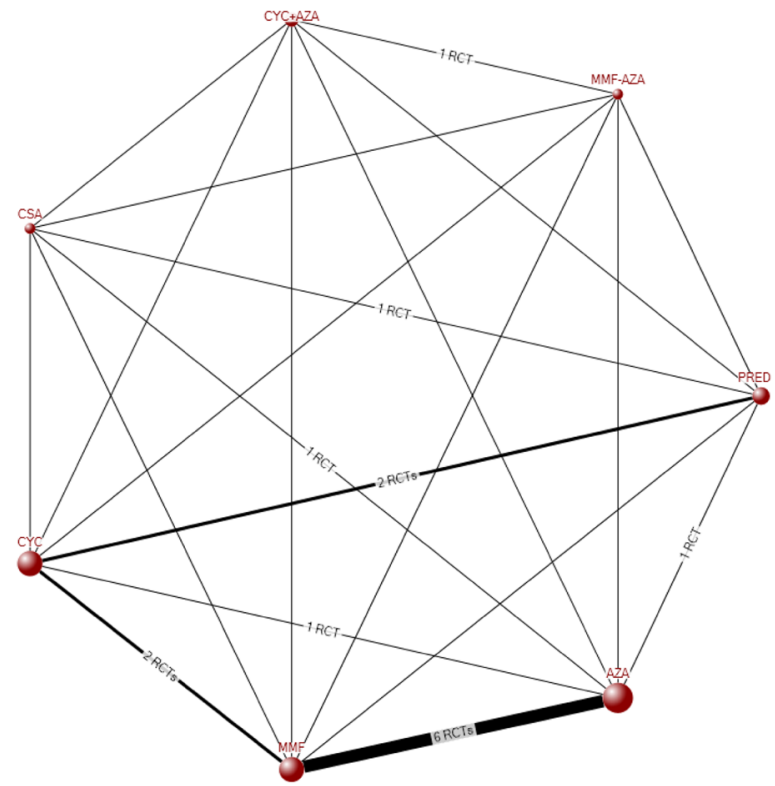

d Bone marrow toxicity

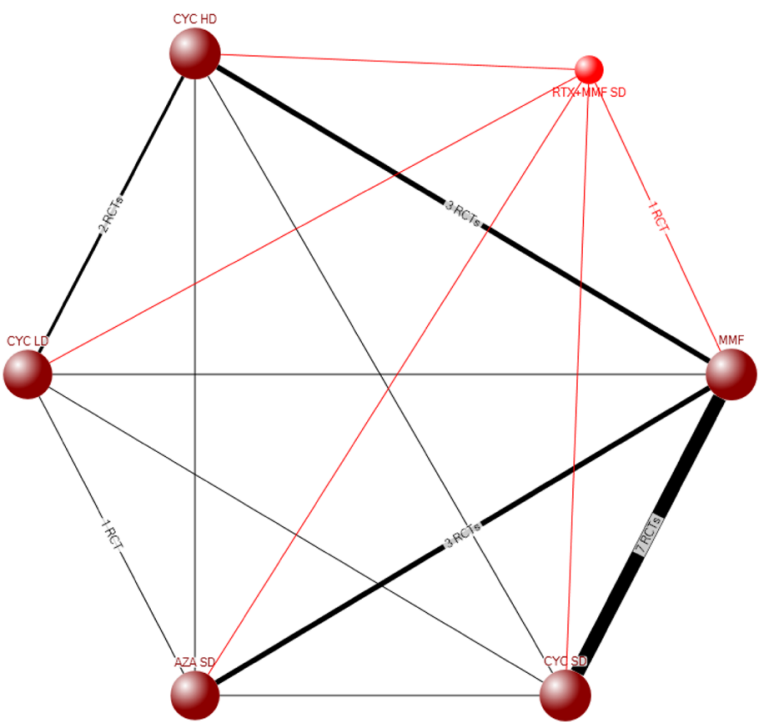

Fig. 2 Network diagrams for composite study outcomes in lupus nephritis, renal remission or renal response (a), renal relapse or renal flare (b), fertility issues (c), and bone marrow toxicity $(\mathbf{d})$. Legend: Each node shows the treatment compared along with the number of RCTs that provide evidence. The size of each node is proportional to the sample size. a shows only the direct evidence, while $\mathbf{b}-\mathbf{d}$ show both direct and indirect evidence. Direct evidence is indicated by lines indicating the number of RCTs providing the evidence and indirect by lines without this information. This was done since there were several direct comparator studies available for $\mathbf{a}$, and addition of connections based on indirect evidence to a would make the network diagram very complex and difficult to visualize and understand 
Table 1 Risk of bias of included studies according to the Cochrane Risk of Bias tool ${ }^{a}$

\begin{tabular}{|c|c|c|c|c|c|c|c|c|}
\hline Author & Year & Randomization & $\begin{array}{l}\text { Allocation } \\
\text { concealment }\end{array}$ & $\begin{array}{l}\text { Blinding of assessor } \\
\text { and/or physician } \\
\text { (for assessment of } \\
\text { objective outcomes) }\end{array}$ & $\begin{array}{l}\text { Blinding of } \\
\text { participants (for } \\
\text { assessment of } \\
\text { subjective outcomes) }\end{array}$ & $\begin{array}{l}\text { Intention } \\
\text { to treat }\end{array}$ & $\begin{array}{l}\text { Free of selective } \\
\text { reporting }\end{array}$ & $\begin{array}{l}\text { Source of } \\
\text { funding }\end{array}$ \\
\hline Austin & 2009 & Low risk & Low risk & High risk & High risk & Unclear risk & Unclear risk & Unclear risk \\
\hline Carette & 1983 & Unclear risk & Unclear risk & High risk & High risk & Low risk & High risk & Low risk \\
\hline Steinberg & 1991 & Low risk & Low risk & Low risk & Low risk & Low risk & Unclear risk & Unclear risk \\
\hline Donadio & 1976 & Unclear risk & Unclear risk & High risk & High risk & Low risk & Unclear risk & Low risk \\
\hline Pohl & 1991 & Unclear risk & Unclear risk & High risk & High risk & Unclear risk & Unclear risk & Unclear risk \\
\hline Wang & 2007 & Unclear risk & Unclear risk & High risk & High risk & High risk & Unclear risk & Unclear risk \\
\hline $\begin{array}{l}\text { Isenberg; An analysis } \\
\text { of ALMS study }\end{array}$ & 2010 & Low risk & Low risk & Low risk & Unclear risk & Low risk & Low risk & Unclear risk \\
\hline Appel (ALMS study) & 2009 & Low risk & Low risk & Low risk & Unclear risk & Low risk & Low risk & Unclear risk \\
\hline Austin & 1986 & Low risk & Unclear risk & Unclear risk & Unclear risk & Unclear risk & Unclear risk & Unclear risk \\
\hline Balletta & 1992 & Unclear risk & Unclear risk & Low risk & Low risk & Low risk & Unclear risk & Low risk \\
\hline Bao & 2008 & Low risk & Low risk & Low risk & Low risk & Low risk & Low risk & Unclear risk \\
\hline Barron & 1982 & High risk & High risk & Low risk & Low risk & High risk & High risk & Low risk \\
\hline Boumpas & 1992 & Unclear risk & Low risk & Low risk & Low risk & Low risk & Low risk & Unclear risk \\
\hline Cade & 1973 & High risk & Unclear risk & Low risk & Low risk & Unclear risk & Unclear risk & Unclear risk \\
\hline Chan & 2000 & Low risk & Unclear risk & Low risk & Low risk & Low risk & Low risk & Unclear risk \\
\hline Chen & 2011 & Low risk & Low risk & Low risk & Low risk & Low risk & Low risk & Unclear risk \\
\hline Clark & 1981 & Unclear risk & Unclear risk & Low risk & Low risk & Low risk & Low risk & Low risk \\
\hline Clark & 1984 & Unclear risk & Unclear risk & Low risk & Low risk & Unclear risk & Unclear risk & Unclear risk \\
\hline Contreras & 2002 & Low risk & Low risk & Low risk & Low risk & Low risk & Low risk & Unclear risk \\
\hline $\begin{array}{l}\text { CYCLOFA-LUNE } \\
\text { Study }\end{array}$ & 2010 & Low risk & Low risk & Low risk & Low risk & Low risk & Low risk & Low risk \\
\hline Derksen & 1988 & Low risk & Unclear risk & Unclear risk & Unclear risk & Unclear risk & Unclear risk & Unclear risk \\
\hline Donadio & 1974 & Low risk & Unclear risk & Low risk & Low risk & Unclear risk & High risk & Low risk \\
\hline Donadio & 1978 & Low risk & Unclear risk & Low risk & Low risk & Unclear risk & Unclear risk & Unclear risk \\
\hline Doria & 1994 & Unclear risk & Unclear risk & Unclear risk & Unclear risk & Unclear risk & Unclear risk & Unclear risk \\
\hline Dyadyk & 2001 & Unclear risk & Unclear risk & Unclear risk & Unclear risk & Unclear risk & Unclear risk & Unclear risk \\
\hline El-Shafey & 2010 & Low risk & Unclear risk & Low risk & Low risk & Low risk & Low risk & Low risk \\
\hline $\mathrm{Fu}$ & 1998 & Low risk & Low risk & Low risk & Low risk & Low risk & High risk & Low risk \\
\hline Ginzler & 2005 & Low risk & Low risk & Low risk & Low risk & Low risk & Low risk & Unclear risk \\
\hline Gourley & 1996 & Low risk & Low risk & Low risk & Low risk & Low risk & Low risk & Low risk \\
\hline $\begin{array}{l}\text { Grootscholten } \\
\text { (Dutch Lupus study) }\end{array}$ & 2006 & Low risk & Low risk & Low risk & Low risk & Low risk & Low risk & Unclear risk \\
\hline Hahn & 1975 & Low risk & Low risk & Low risk & Low risk & Low risk & Low risk & Low risk \\
\hline Hong & 2007 & Unclear risk & Unclear risk & Unclear risk & Unclear risk & Unclear risk & Unclear risk & Unclear risk \\
\hline Houssiau & 2002 & Low risk & Unclear risk & Low risk & Low risk & Low risk & Low risk & Low risk \\
\hline Lewis & 1992 & Low risk & Unclear risk & Low risk & Low risk & Low risk & Low risk & Low risk \\
\hline $\mathrm{Li}$ & $2009 a$ & Low risk & Low risk & Low risk & Low risk & Low risk & Low risk & Unclear risk \\
\hline $\mathrm{Li}$ & $2009 b$ & Unclear risk & Unclear risk & Low risk & Low risk & Low risk & Low risk & Low risk \\
\hline Lui & 1997 & Unclear risk & Unclear risk & Unclear risk & Unclear risk & Unclear risk & Unclear risk & Unclear risk \\
\hline LUNAR Study & 2012 & Low risk & Unclear risk & Low risk & Low risk & Low risk & Low risk & Unclear risk \\
\hline $\begin{array}{l}\text { MAINTAIN Nephritis } \\
\text { Study }\end{array}$ & 2010 & Low risk & Unclear risk & Low risk & Low risk & Low risk & Low risk & Low risk \\
\hline Mitwalli & 2011 & Unclear risk & Unclear risk & Unclear risk & Unclear risk & Unclear risk & Unclear risk & Unclear risk \\
\hline
\end{tabular}


Table 1 Risk of bias of included studies according to the Cochrane Risk of Bias tool ${ }^{a}$ (Continued)

\begin{tabular}{|c|c|c|c|c|c|c|c|c|}
\hline Mok & 2009 & Unclear risk & Unclear risk & Unclear risk & Unclear risk & Unclear risk & Unclear risk & Unclear risk \\
\hline Moroni & 2004 & Low risk & Low risk & Low risk & Low risk & Low risk & Low risk & Unclear risk \\
\hline Mulic-Basic & 2008 & Unclear risk & Unclear risk & Unclear risk & Unclear risk & Unclear risk & Unclear risk & Unclear risk \\
\hline My-Lupus Study & 2010 & Unclear risk & Unclear risk & Unclear risk & Unclear risk & Unclear risk & Unclear risk & Unclear risk \\
\hline Ong & 2005 & Low risk & Low risk & Low risk & Low risk & Low risk & Low risk & Unclear risk \\
\hline Sabry & 2009 & High risk & High risk & Low risk & Low risk & Low risk & Low risk & Low risk \\
\hline Sesso & 1994 & Unclear risk & Unclear risk & High risk & Low risk & Low risk & Unclear risk & Low risk \\
\hline Steinberg & 1971 & Low risk & Low risk & Low risk & Low risk & Low risk & Low risk & Unclear risk \\
\hline Sundel/Sandel & 2008 & Unclear risk & Unclear risk & Unclear risk & Unclear risk & Unclear risk & Unclear risk & Unclear risk \\
\hline Wallace & 1998 & Unclear risk & Unclear risk & Unclear risk & Unclear risk & Unclear risk & Unclear risk & Unclear risk \\
\hline Yee & 2004 & Low risk & Unclear risk & Low risk & Low risk & Low risk & High risk & Low risk \\
\hline $\mathrm{Li}$ & 2012 & Unclear risk & Unclear risk & High risk & High risk & High risk & Unclear risk & Low risk \\
\hline Yap & 2012 & Unclear risk & Unclear risk & High risk & High risk & High risk & Unclear risk & Unclear risk \\
\hline Stoenoiu & 2012 & Unclear risk & Unclear risk & Unclear risk & Unclear risk & High risk & Unclear risk & Unclear risk \\
\hline Chen & 2012 & Low risk & Low risk & High risk & High risk & High risk & Unclear risk & Unclear risk \\
\hline $\begin{array}{l}\text { Arends (long term } \\
\text { FU of Dutch } \\
\text { Lupus study) }\end{array}$ & 2012 & Low risk & Low risk & Low risk & Low risk & Low risk & Low risk & Unclear risk \\
\hline $\begin{array}{l}\text { Sundel (report } \\
\text { of induction and } \\
\text { maintenance phases } \\
\text { of ALMS study) }\end{array}$ & 2012 & Low risk & Low risk & Low risk & Unclear risk & Low risk & Low risk & Unclear risk \\
\hline $\begin{array}{l}\text { Walsh (post-hoc } \\
\text { subgroup analysis } \\
\text { of ALMS) }\end{array}$ & 2013 & Low risk & Low risk & Low risk & Unclear risk & Low risk & Low risk & Unclear risk \\
\hline Petrl & 2010 & Low risk & Unclear & High risk & High risk & Low risk & Unclear risk & Low risk \\
\hline Zeher & 2011 & Low risk & Low risk & High risk & High risk & High risk & Unclear risk & High risk \\
\hline Dooley & 2011 & Unclear risk & Unclear risk & Unclear risk & Unclear risk & High risk & Unclear risk & High risk \\
\hline
\end{tabular}

Radhakrishnan 2010 was a pooled analyses of two studies, and Mok 2001, Hu 2002, and Wang 2008 [33-36] were observational studies used in the Cochrane Review; therefore, risk of bias could not be assessed for these studies

a Higgins JP, Altman DG, Sterne JA. Chapter 8: Assessing risk of bias in included studies. Cochrane Handbook for Systematic Reviews of Interventions Version 5.1.0 (updated March 2011). The Cochrane Collaboration, 2011. Available from http://handbook.cochrane.org/. In: Higgins JP, Green S, eds 2011

effectiveness of one immunosuppressive drug vs. another and immunosuppressive drugs vs. corticosteroids. Bayesian MTC meta-analysis using a binomial likelihood model was conducted using WinBUGS software (MRC Biostatistics Unit, Cambridge, UK) which allows inclusion of data from multi-arm trials $[25,26]$ We conduced randomeffects NMA and assessed model fit and the choice of model (random vs. fixed effects) based on the assessment of the deviance information criterion (DIC) and the comparison of residual deviance to the number of unconstrained data points $[25,27]$.

We assigned vague priors, such as $\mathrm{N}\left(0,100^{2}\right)$ for basic parameters throughout [25] and informative priors for the variance parameter based on Turner et al. [28]. We evaluated the model diagnostics including trace plots and the Brooks-Gelman-Rubin statistic to ensure model convergence $[25,29]$. We fit three chains in WinBUGS for each analysis, with 40,000 iterations, and a burn-in of 40,000 iterations $[29,30]$
Both MTC and traditional meta-analysis require studies to be sufficiently similar in order to pool their results. We investigated heterogeneity, where warranted, with subgroup analyses and meta-regressions $[26,31]$. We examined consistency-inconsistency plots for evidence of inconsistency, and chose the appropriate model for our analyses. We obtained point estimates using odds ratios (OR) and $95 \%$ credible intervals (CrI) using Markov Chain Monte Carlo (MCMC) methods. Transformation of the OR to relative risk (RR) and risk difference was done to allow ease for interpretation for clinicians and patients. The quality of evidence was assessed as recommended in a recent study [32].

Sensitivity analysis was performed by limiting analyses to partial/complete remission rather than combining this with renal response for the composite renal remission/response. We constructed staircase diagrams, another pictorial way to see comparisons of various treatments to each other. 
Rankograms were constructed to model the probabilities of the treatment rankings, representing the best to the last ranks.

\section{Results}

\section{Study characteristics}

Sixty-five studies met inclusion and exclusion criteria that included CYC, MMF, AZA, calcineurin inhibitors (cyclosporine, tacrolimus), rituximab, corticosteroids, plasmapharesis, or leflunomide (Fig. 1). The Additional file 2 shows the search strategy. An additional file shows the PRISMA checklist (see Additional file 3).

Network diagrams for all outcomes are shown in Fig. 2. Most studies (88\%) compared induction or induction and maintenance regimens. An additional file shows this in more detail (see Additional file 4). The study sample size ranged from 10 to 370 . Of these, $32 \%$ of the studies were conducted in the USA and $43 \%$ were multicenter.

A detailed risk of bias using the Cochrane risk of bias tool is provided in Table 1. Randomization was low-risk in $56 \%$, unclear in $39 \%$ and high-risk in $5 \%$ (Table 1). Most trials were low-risk for blinding of assessor (59\%), blinding of participant (54\%), intention to treat (57\%). On the other hand, only $38 \%$ of trials were low-risk for allocation concealment and it was unclear in $59 \%$.
Although some clinical heterogeneity was detected between trials overall, we did not notice any clinically significant systematic differences in patient populations or disease stages between various medications.

\section{Treatment efficacy: complete/partial renal remission/response}

Thirty-seven trials with 2697 patients provided data for the composite outcome, partial or complete renal remission or renal response (two trials were excluded since they had variable duration of treatments based on response to initial treatment, also associated with high standard errors and wide CrI, leading to problems with convergence of the model when included). There were 34 two-arm and three three-arm trials. Table 2 shows only the significant odds ratios, relative risk and risk differences only, and an additional file shows all comparisons in more detail (see Additional file 5). CYC, MMF, CSA, and TAC were superior to corticosteroids alone in achieving renal remission/response (Table 2). CYC low dose (LD) was less likely than MMF, TAC, CSA, and CYC and CYC HD less likely than MMF and CSA to achieve renal remission/response. CSA was more likely than plasmapharesis and azathioprine to achieve renal remission/response (Table 2). The quality of evidence

Table 2 Significant differences ${ }^{a}$ between treatments of lupus nephritis for a composite end-point of renal remission or renal response (includes partial remission, complete remission and renal response)

\begin{tabular}{|c|c|c|c|c|}
\hline Treatment & Reference & Odds ratio $(95 \% \mathrm{Crl})$ & Relative risk (95\% Crl) & Risk difference \% (95\% Crl) \\
\hline$\overline{C Y C}$ & PRED & $2.35(1.28,4.23)$ & $1.60(1.60,2.24)$ & $0.21(0.06,0.34)$ \\
\hline MMF & & $3.26(1.57,6.72)$ & $1.82(1.82,2.60)$ & $0.28(0.11,0.44)$ \\
\hline TAC & & $2.51(1.11,5.76)$ & $1.64(1.64,2.44)$ & $0.22(0.03,0.41)$ \\
\hline CSA & & $5.69(2.02,17.61)$ & $2.15(2.15,3.18)$ & $0.40(0.17,0.58)$ \\
\hline CYC LD & CYC & $0.32(0.10,0.89)$ & $0.51(0.51,0.95)$ & $-0.26(-0.45,-0.03)$ \\
\hline CYC LD & MMF & $0.23(0.08,0.61)$ & $0.45(0.45,0.81)$ & $-0.34(-0.53,-0.12)$ \\
\hline CYC HD & & $0.40(0.20,0.74)$ & $0.65(0.65,0.89)$ & $-0.22(-0.38,-0.07)$ \\
\hline CSA & AZA & $3.20(1.04,10.19)$ & $1.53(1.53,2.39)$ & $0.26(0.01,0.47)$ \\
\hline CYC LD & TAC & $0.30(0.09,0.91)$ & $0.50(0.50,0.95)$ & $-0.28(-0.50,-0.02)$ \\
\hline PLASMA & CSA & $0.19(0.04,0.92)$ & $0.49(0.49,0.97)$ & $-0.38(-0.66,-0.02)$ \\
\hline CYC LD & & $0.13(0.03,0.52)$ & $0.38(0.38,0.76)$ & $-0.45(-0.69,-0.15)$ \\
\hline CYC HD & & $0.23(0.06,0.72)$ & $0.55(0.55,0.87)$ & $-0.33(-0.57,-0.08)$ \\
\hline CYC HD & & $0.23(0.06,0.72)$ & $0.55(0.55,0.87)$ & $-0.33(-0.57,-0.08)$ \\
\hline \multirow[t]{2}{*}{ Random-effects model } & Residual deviance & & 82.2 vs. 77 data points & \\
\hline & Deviance information criteria & & 389.332 & \\
\hline \multirow[t]{2}{*}{ Fixed-effects model } & Residual Deviance & & 88.8 vs. 77 data points & \\
\hline & Deviance information criteria & & 390.488 & \\
\hline
\end{tabular}

Based on 37 RCTs with 2697 patients: 35 two-arm trials and three three-arm trials

${ }^{a}$ For all comparisons of treatments of lupus nephritis for renal remission/response please see Appendix 5

For absolute rates for events used for calculation of risk difference, please see Appendix 6

$O R$ odds ratio, $R R$ relative risk, $R D$ risk difference, $C r l$ credible interval, $C Y C$ cyclophosphamide, MMF mycophenolate mofetil, CSA cyclosporine, TAC tacrolimus, $L E F$ leflunomide, PRED prednisone, prednisolone or methylprednisolone, $A Z A$ azathioprine, $R T X$ rituximab, $H D$ high dose, $L D$ low dose, when not specified, it indicates standard dose Significant odds ratios are in italics

The odds ratios were transformed to relative risk (RR) and risk difference was done to allow ease for interpretation for clinicians and patients 
was rated as moderate (downgraded for imprecision). Absolute event rates ranged from 28 to $75 \%$ and are shown in more detail in an additional file (see Additional file 6).

\section{Treatment failure: renal relapse/renal flare}

Thirteen studies with 1,108 patients provided data; 11 were two-arm and two were three-arm studies. MMF and $\mathrm{CYC}$ were associated with a lower rate of renal relapse/flare compared to PRED and MMF was associated with a lower rate of renal relapse/flare than AZA (Table 3). The quality of evidence was rated as moderate (downgraded for imprecision). The event rates ranged from 14 to $49 \%$ and are shown in more detail in an additional file (see Additional file 6).

\section{Amenorrhea/ovarian failure}

Eight RCTs with 839 patients provided data; seven were two-arm and one trial was a three-arm trial. CYC was more likely than MMF and PRED to be associated with amenorrhea/ovarian failure (Table 4). CYC LD was associated with higher risk of amenorrhea/ovarian failure than MMF. The quality of evidence was rated as moderate (downgraded for imprecision). Absolute event rates ranged from 8 to $61 \%$ and are shown in more detail in an additional file (see Additional file 6).

Bone marrow toxicity: cytopenia (including leucopenia) Sixteen trials provided data on 2257 patients: 15 were two-arm and one was a three-arm trial. Compared to MMF, several immunosuppressive drugs were associated with a higher risk of cytopenia: CYC, AZA, CYC LD,

Table 3 Comparison of all lupus nephritis treatments for a composite of renal relapse or renal flare

\begin{tabular}{|c|c|c|c|c|}
\hline Treatment & Reference & Odds ratio $(95 \% \mathrm{Crl})$ & Relative risk (95 \% Crl) & Risk difference \% (95\% Crl) \\
\hline$\overline{A Z A}$ & PRED & $0.33(0.08,1.23)$ & $0.50(0.17,1.12)$ & $-0.24(-0.47,0.05)$ \\
\hline MMF & & $0.17(0.04,0.67)$ & $0.29(0.09,0.78)$ & $-0.34(-0.53,-0.09)$ \\
\hline CYC & & $0.18(0.05,0.58)$ & $0.31(0.11,0.71)$ & $-0.33(-0.51,-0.12)$ \\
\hline CSA & & $0.24(0.03,1.60)$ & $0.39(0.07,1.26)$ & $-0.29(-0.52,0.11)$ \\
\hline$C Y C+A Z A$ & & $0.32(0.05,1.67)$ & $0.48(0.10,1.29)$ & $-0.25(-0.50,0.12)$ \\
\hline MMF-AZA & & $0.41(0.03,5.46)$ & $0.58(0.07,1.83)$ & $-0.20(-0.51,0.36)$ \\
\hline MMF & AZA & $0.51(0.32,0.87)$ & $0.59(0.38,0.90)$ & $-0.10(-0.20,-0.02)$ \\
\hline CYC & & $0.55(0.24,1.26)$ & $0.62(0.32,1.21)$ & $-0.09(-0.260 .03)$ \\
\hline CSA & & $0.74(0.18,2.95)$ & $0.80(0.23,2.01)$ & $-0.04(-0.23,0.23)$ \\
\hline$C Y C+A Z A$ & & $0.95(0.27,3.09)$ & $0.96(0.34,2.11)$ & $-0.01(-0.19,0.24)$ \\
\hline MMF-AZA & & $1.23(0.14,12.15)$ & $1.16(0.19,3.76)$ & $0.04(-0.25,0.53)$ \\
\hline CYC & MMF & $1.06(0.44,2.58)$ & $1.05(0.51,2.29)$ & $0.01(-0.13,0.12)$ \\
\hline CSA & & $1.43(0.32,6.17)$ & $1.33(0.36,3.81)$ & $0.04(-0.12,0.35)$ \\
\hline$C Y C+A Z A$ & & $1.84(0.51,6.00)$ & $1.62(0.56,3.85)$ & $0.08(-0.07,0.35)$ \\
\hline MMF-AZA & & $2.39(0.27,23.30)$ & $1.94(0.31,6.94)$ & $0.13(-0.12,0.63)$ \\
\hline CSA & CYC & $1.35(0.27,6.52)$ & $1.27(0.31,3.86)$ & $0.04(-0.14,0.37)$ \\
\hline$C Y C+A Z A$ & & $1.72(0.44,6.37)$ & $1.53(0.49,3.83)$ & $0.08(-0.09,0.3)$ \\
\hline MMF-AZA & & $2.24(0.24,23.15)$ & $1.85(0.28,6.46)$ & $0.13(-0.13,0.63)$ \\
\hline$C Y C+A Z A$ & CSA & $1.27(0.20,8.09)$ & $1.20(0.30,5.35)$ & $0.03(-0.29,0.35)$ \\
\hline MMF-AZA & & $1.67(0.13,25.89)$ & $1.43(0.19,8.68)$ & $0.08(-0.31,0.62)$ \\
\hline MMF-AZA & $\mathrm{CYC}+\mathrm{AZA}$ & $1.30(0.22,9.13)$ & $1.20(0.28,3.68)$ & $0.04(-0.01,0.46)$ \\
\hline \multirow[t]{2}{*}{ Random-effects model } & Residual deviance & 32.06 vs. 28 data points & & \\
\hline & Deviance information criteria & 146.869 & & \\
\hline \multirow[t]{2}{*}{ Fixed-effects model } & Residual deviance & 34.31 vs. 28 data points & & \\
\hline & Deviance information criteria & 147.018 & & \\
\hline
\end{tabular}

Based on 13 RCTs with 1108 patients: 11 two-arm trials and two three-arm trials

Significant odds ratios are in italics

For absolute rates for events used for calculation of risk difference, please see Appendix 6

$O R$ odds ratio, $R R$ relative risk, $R D$ risk difference, $C r l$ credible interval, $C Y C$ cyclophosphamide, MMF mycophenolate mofetil, $C S A$ cyclosporine, $T A C$ tacrolimus, $L E F$ leflunomide, $P R E D$ prednisone, prednisolone or methylprednisolone, $A Z A$ azathioprine, $R T X$ rituximab

CYC + AZA CYC with AZA, MMF-AZA MMF followed by AZA

Merged doses for PRED and CYC and comparing only between treatments. We did not lose any study, but it is a limitation of this analysis 
Table 4 Comparison of all lupus nephritis treatments for fertility issues (amenorrhea or ovarian failure)

\begin{tabular}{lllll}
\hline Treatment & Reference & OR $(95 \% \mathrm{Crl})$ & $\mathrm{RR}(95 \% \mathrm{Crl})$ & $\mathrm{RD} \%(95 \% \mathrm{Crl})$ \\
\hline CYC & PRED & $3.81(1.26,14.31)$ & $2.64(1.18,7.51)$ & $0.25(0.04,0.47)$ \\
MMF & & $0.48(0.09,3.04)$ & $0.52(0.11,2.58)$ & $-0.07(-0.23,0.13)$ \\
AZA & & $2.74(0.26,28.27)$ & $2.12(0.30,8.18)$ & $0.17(-0.15,0.65)$ \\
CYC LD & CYC & $9.06(0.63,121.50)$ & $3.79(0.68,11.71)$ & $0.45(-0.06,0.81)$ \\
MMF & & $0.13(0.03,0.41)$ & $0.20(0.05,0.56)$ & $-0.31(-0.47,-0.16)$ \\
AZA & & $0.69(0.09,5.08)$ & $0.79(0.14,1.95)$ & $-0.08(-0.37,0.36)$ \\
CYC LD & MMF & $2.23(0.23,23.20)$ & $1.46(0.34,2.71)$ & $0.19(-0.27,0.55)$ \\
AZA & & $5.25(0.78,54.39)$ & $3.68(0.81,19.08)$ & $0.22(-0.02,0.68)$ \\
CYC LD & $16.35(1.97,247.70)$ & $6.49(1.72,30.95)$ & $0.50(0.06,0.87)$ \\
CYC LD & AZA & $3.20(0.55,21.24)$ & $1.69(0.68,6.12)$ & $0.22(-0.11,0.60)$ \\
Random-effects model & Residual deviance & 15.93 vs. 17 data points & & \\
& Deviance information criteria & 72.233 & & \\
Fixed-effects model & Residual deviance & 16.16 vs. 17 data points & & \\
& Deviance information criteria & 72.069 & & \\
\hline
\end{tabular}

Based on eight RCTs with 839 patients: seven two-arm trials and one three-arm trial

Significant odds ratios are in italics

For absolute rates for events used for calculation of risk difference, please see Appendix 6

$O R$ odds ratio, $R R$ relative risk, $R D$ risk difference, $C r l$ credible interval, $H D$ high dose, $L D$ low dose; when not specified, it indicates standard dose, $C Y C$ cyclophosphamide, $M M F$ mycophenolate mofetil, CSA cyclosporine, TAC tacrolimus, LEF leflunomide, PRED prednisone, prednisolone or methylprednisolone, AZA azathioprine, RTX rituximab The odds ratios were transformed to relative risk (RR) and risk difference was done to allow ease for interpretation for clinicians and patients

and CYC HD (Table 5). The quality of evidence was rated as moderate (downgraded for imprecision). Absolute event rates ranged from 7 to $30 \%$ and are shown in more detail in an additional file (see Additional file 6).

\section{Sensitivity analyses limited to partial or complete renal remission only}

An additional file shows in detail the odds ratios for comparisons of immunosuppressive drugs and corticosteroids in lupus nephritis for partial/complete remission, a sensitivity analysis (renal response excluded from the composite outcome; see Additional file 7). Results were similar to the main analyses, with minor exceptions. Thus, most observations from the main analysis were confirmed in this sensitivity analysis.

\section{Rankograms and staircase diagrams}

Figure 2 shows the Rankograms for various treatments for the outcomes of interest. Among the top ranked were CSA for renal remission/response, prednisone for renal relapse/flare, CYC for ovarian failure/amenorrhea and CYC for bone marrow toxicity (Fig. 3). An additional file shows in detail comparisons of various treatments to each other for each outcome, another way to visualize the key comparisons between treatments (see Additional file 8).

\section{Discussion}

In this systematic review and NMA of outcomes in patients with lupus nephritis, we made several important observations. Results of this study are of great value to both medical and patient communities, given the growing and renewed focus on patient-centered education and outcomes. We directly compared benefits and risks of medications that are used to treat SLE nephritis. The information presented here served as the foundation for a decision-aid tool that can be easily understood that is being tested in a randomized trial, currently underway. Given the novel NMA methodology and our ability to perform indirect comparisons using the NMA, several of our findings are new and merit further discussion.

We noted differences between immunosuppressive drugs regarding renal remission/response. Interestingly, high-dose CYC was significantly less effective than MMF in leading to renal remission/response with a relative risk of 0.65 and an odds ratio of 0.40 . This is an interesting finding and is consistent with the trial data from Ginzler et al. that found that MMF was superior to IV CYC in inducing complete renal remission [37].

We also found that CYC LD (which includes EUROlupus regimen) was inferior to MMF, CSA and CYC standard dose for renal remission/response. We realize that not all trials are same; however, the superiority of these commonly used immunosuppressive drugs to CYC LD is worth some discussion. This benefit of using low-dose CYC must be weighed against the potential harms of using MMF, CSA or CYC standard dose in a patient with lupus nephritis. Several other differences we noted might be of interest to clinicians and patients when making treatment decisions for lupus nephritis. 
Table 5 Comparison of all lupus nephritis treatments for bone marrow toxicity (cytopenia including leucopenia)

\begin{tabular}{|c|c|c|c|c|}
\hline Treatment & Reference & OR $(95 \% \mathrm{Crl})$ & RR (95\% Crl) & RD \% (95 \% Crl) \\
\hline CYC SD & \multirow[t]{5}{*}{ MMF } & $2.16(1.14,4.03)$ & $1.99(1.13,3.37)$ & $0.07(0.01,0.16)$ \\
\hline AZA SD & & $2.42(1.01,7.07)$ & $2.19(1.01,5.06)$ & $0.09(0.00,0.27)$ \\
\hline CYC LD & & $5.31(1.26,24.65)$ & $4.03(1.23,10.11)$ & $0.22(0.02,0.56)$ \\
\hline CYC HD & & $5.36(1.95,18.08)$ & $4.06(1.81,8.96)$ & $0.22(0.07,0.48)$ \\
\hline$R T X+M M F S D$ & & $3.50(0.61,23.14)$ & $2.95(0.63,9.43)$ & $0.14(-0.03,0.56)$ \\
\hline AZA SD & \multirow[t]{4}{*}{ CYC SD } & $1.11(0.39,4.03)$ & $1.09(0.44,3.07)$ & $0.01(-0.11,0.22)$ \\
\hline CYC LD & & $2.45(0.51,13.07)$ & $2.01(0.56,5.91)$ & $0.15(-0.08,0.50)$ \\
\hline CYC HD & & $2.47(0.77,9.99)$ & $2.03(0.80,5.38)$ & $0.15(-0.04,0.43)$ \\
\hline $\mathrm{RTX}+\mathrm{MMF} \mathrm{SD}$ & & $1.63(0.26,11.77)$ & $1.49(0.30,5.31)$ & $0.07(-0.13,0.49)$ \\
\hline CYC LD & \multirow[t]{3}{*}{ AZA SD } & $2.16(0.42,10.83)$ & $1.80(0.49,5.39)$ & $0.13(-0.12,0.47)$ \\
\hline CYC HD & & $2.20(0.56,8.96)$ & $1.83(0.65,5.16)$ & $0.13(-0.10,0.40)$ \\
\hline $\mathrm{RTX}+\mathrm{MMF} \mathrm{SD}$ & & $1.43(0.18,11.42)$ & $1.33(0.23,5.33)$ & $0.05(-0.21,0.48)$ \\
\hline CYC HD & \multirow[t]{2}{*}{ CYC LD } & $1.01(0.29,3.75)$ & $1.01(0.46,2.74)$ & $0.00(-0.28,0.24)$ \\
\hline $\mathrm{RTX}+\mathrm{MMF} \mathrm{SD}$ & & $0.66(0.06,7.15)$ & $0.74(0.12,3.78)$ & $-0.07(-0.48,0.40)$ \\
\hline$R T X+M M F S D$ & CYC HD & $0.65(0.08,5.36)$ & $0.73(0.13,2.81)$ & $-0.08(-0.41,0.37)$ \\
\hline \multirow[t]{2}{*}{ Random-effects model } & \multicolumn{2}{|l|}{ Residual deviance } & \multicolumn{2}{|l|}{31.43 vs. 33 data points } \\
\hline & \multicolumn{2}{|l|}{ Deviance information criteria } & \multicolumn{2}{|l|}{165.076} \\
\hline \multirow[t]{2}{*}{ Fixed-effects model } & \multicolumn{2}{|l|}{ Residual deviance } & \multicolumn{2}{|l|}{38.78 vs. 33 data points } \\
\hline & \multicolumn{2}{|l|}{ Deviance information criteria } & \multicolumn{2}{|l|}{167.984} \\
\hline
\end{tabular}

Based on 16 RCTs with 2257 patients: 15 two-arm trials and one three-arm trial Significant odds ratios are in italics

For absolute rates for events used for calculation of risk difference, please see Appendix 6

$O R$ odds ratio, $R R$ relative risk, $R D$ risk difference, $C r l$ credible interval, $H D$ high dose, $L D$ low dose; when not specified, it indicates standard dose, $C Y C$ cyclophosphamide, $M M F$ mycophenolate mofetil, CSA cyclosporine, TAC tacrolimus, LEF leflunomide, PRED prednisone, prednisolone or methylprednisolone, AZA azathioprine, RTX rituximab MMF-AZA, MMF followed by AZA

RTX + MMF, RTX combined with MMF

Estimates for LEF HD were obtained from data from only one study and were therefore imprecise

The odds ratios were transformed to relative risk (RR) and risk difference was done to allow ease for interpretation for clinicians and patients

We confirmed that immunosuppressive drugs were more effective than corticosteroids alone (most often prednisone or prednisolone) for renal remission/response by two to four times, that is, CYC, MMF, CSA, and tacrolimus led to renal remission significantly more often than corticosteroids alone. The lower risk of renal relapse or flare with MMF and CYC compared to corticosteroids alone is also supportive of this finding and not surprising. Other immunosuppressives (AZA, CSA, CYCAZA, MMF-AZA) also seemed to be possibly more effective than corticosteroids alone for preventing renal relapse/flare, but did not reach significance.

Significant differences were found in the risk of amenorrhea/ovarian failure (fertility issues). These are important findings, since a disproportionate number of young women are affected by lupus. An increased risk of amenorrhea/ovarian failure was seen with CYC compared to MMF and PRED, which confirms a long-known clinical observation, but now provides estimates of the comparative risk. Ovarian failure is an important discussion point during lupus treatment decision-making in young women, especially when the use of CYC is considered.
We found that CYC SD, CYC LD, CYC HD, and AZA had two to five times higher risk of cytopenia than MMF. Our comprehensive NMA provided a robust treatment estimates for these comparisons that can be shared with patients in a more understandable format at the time of treatment decision-making for lupus nephritis in regular clinical care.

Our study has several limitations. Meta-analyses are observational studies and therefore can have limitations of any observational study. Heterogeneity is an issue with all meta-analyses, including NMA. We assessed for clinical heterogeneity of studies prior to conducting the analyses, with the help of two clinicians (including a rheumatologist and a lupus expert) who assessed for systematic differences between study populations and disease stage by medications regimens. We noted some clinical heterogeneity between trials, but did not find major systematic differences by the type of medication used. We acknowledge that no two clinical trials are alike. This applies to our NMA as well, and therefore, the results should be interpreted with some caution. Another study limitation may be that we searched two databases. 


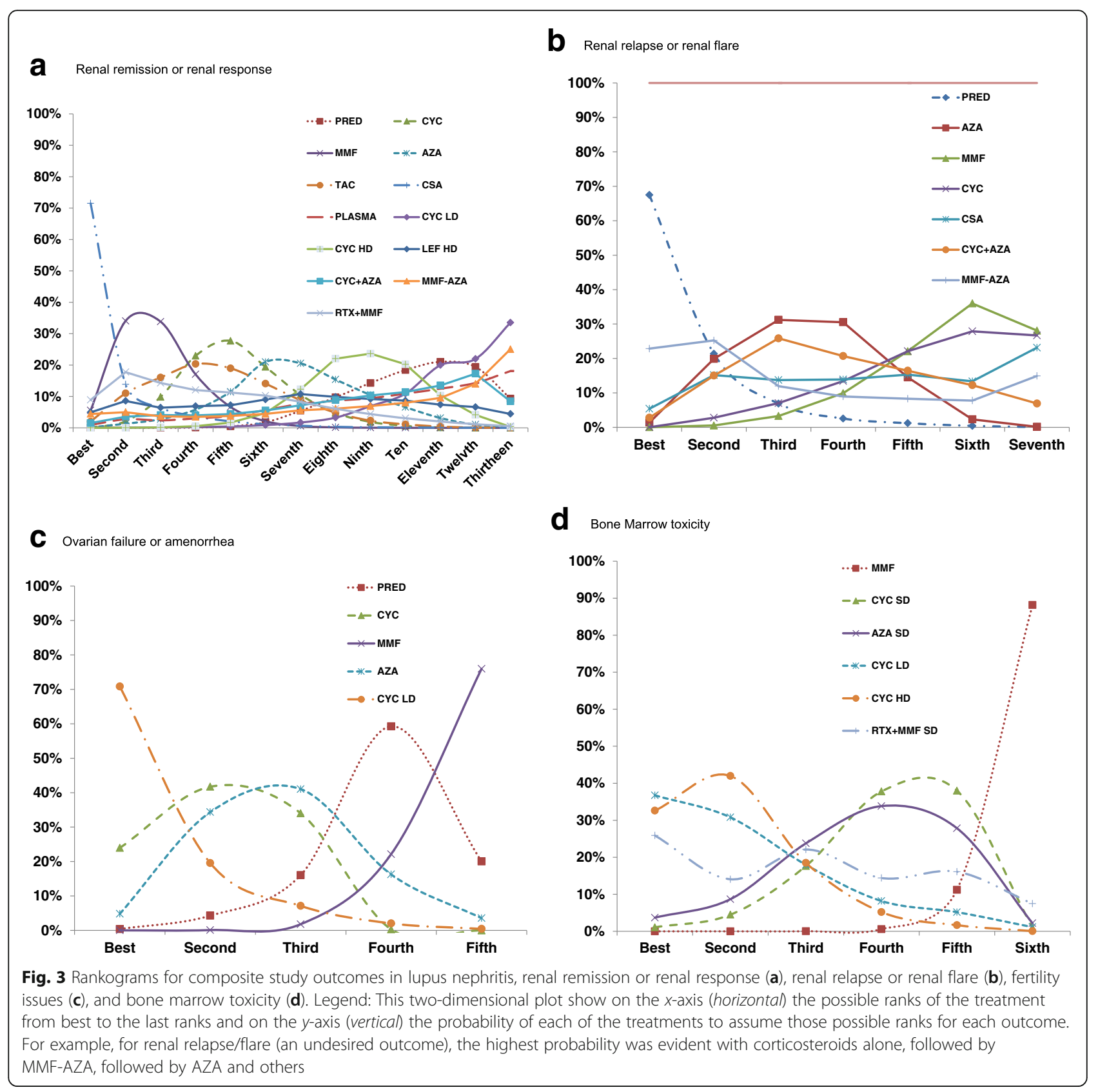

The NMA incorporates both direct and indirect comparisons. As can be seen from the network diagrams for these analyses, for some outcomes assessed in this study, direct comparisons were fewer, which indicates that most evidence came from indirect comparisons; addition of data from direct comparator trials in future NMA would increase the confidence in these findings. Due to multiple NMA comparisons performed, some findings may be resulting from chance. However, we think that type II error, i.e., missing important differences due to small sample size, is a bigger concern, since most included trials for this rare condition, were of small size.
For comparisons with large relative effects (odds or risk ratios), one should keep in mind the underlying rates, which varied between outcomes and were very low for some composite outcomes. In these cases, the absolute difference between treatments is still small, even though relative effect may be five or ten times, or 0.05 times. Comparisons with wider confidence intervals must be interpreted with caution, since these indicate small study numbers, or a lower confidence in the certainty of the estimate. It is possible (and even likely sometimes) that addition of more data from studies in the future may change these estimates. 


\section{Conclusions}

This systematic review and NMA provides the most current, comprehensive comparison of immunosuppressive medications and corticosteroids used to treat lupus nephritis. These findings must be interpreted considering between study heterogeneity. This comparative effectiveness research provides estimates of key benefits and harms that would be of interest to both physicians and their patients. The state-of-the-art methodology used here should be duplicated for other diseases where physicians and patients will benefit from comparisons of commonly used treatments.

\section{Additional files}

Additional file 1: Detailed definitions of efficacy outcomes and medication doses. (DOCX $22 \mathrm{~kb}$ )

Additional file 2: A. PubMed search strategy for updating the American College of Rheumatology (ACR) lupus treatment guideline and the Cochrane library searches. B. Second Search strategy to identify any lupus trial for side effects of medications in PubMed and Scopus databases (data abstracted, but not used due to scant data and the possibility of heterogeneity in patient population, i.e., lupus nephritis vs. lupus with all other manifestations). (DOC $25 \mathrm{~kb}$ )

Additional file 3: PRISMA NMA checklist of items to include when reporting a systematic review involving a network meta-analysis. (DOCX $97 \mathrm{~kb}$ )

Additional file 4: Characteristics of included studies. (DOCX $41 \mathrm{~kb}$ )

Additional file 5: All Comparisons of various treatments for the composite end-point of renal remission or renal response (includes partial remission/complete remission/renal response). (DOCX 28 kb)

Additional file 6: Event rates for the outcomes of interest. (DOCX $19 \mathrm{~kb}$ ) Additional file 7: Sensitivity analysis including partial/complete renal remission only. (DOCX 29 kb)

Additional file 8: Staircase diagrams for key outcomes. (DOCX 26 kb)

\section{Abbreviations}

NMA: Network meta-analysis; OR: Odds ratio; Crl: Credible interval; MTC: Mixed treatment comparison; AHRQ: Agency for Healthcare Research and Quality; UAB: University of Alabama at Birmingham; RCTs: Randomized controlled trials; CCTs: Controlled clinical trials; PRED: Corticosteroids, including prednisone; CYC: Cyclophosphamide; MMF: Mycophenolate mofetil; AZA: Azathioprine; CSA: Cyclosporine; TAC: Tacrolimus; RTX: Rituximab; LD: Low-dose/duration; HD: High-dose/duration; SD: Standard-dose/duration; IV: Intravenously; PO: Orally;

PLASMA: Plasmapharesis

\section{Acknowledgements}

This research was made possible by Grant from Patient Centered Outcomes Research Institute (PCORI; Grant Number CE-1304-6631), and its contents are solely the responsibility of the authors and do not necessarily represent the official views of the Patient- Centered Outcomes Research Institute. We thank the three librarians, Tamara Rader (TR) from the Cochrane Collaboration, Janet Joyce (J.J.) from the American College of Rheumatology and Carolynn Holmes $(\mathrm{CH})$ from the University of Alabama at Birmingham (UAB), for performing the literature searches. We thank the following people at UAB; Aseem Bharat $(A B)$ and Ana Oliveira $(A O)$ for screening titles and abstracting data; Amy Mudano (AM) for data programming; and Nipam Shah for a careful review of the final manuscript. We thank Drs. Jennifer Grossman and Bevra Hahn of the University of California at Los Angeles, Los Angeles, CA of the ACR lupus nephritis guidelines team, for sharing their evidence report tables with us, and Dr. Grossman for examining the included studies for similarity.

\section{Funding}

This research was made possible by a Grant from the Patient Centered Outcomes Research Institute (PCORI; Grant Number CE-1304-6631), and its contents are solely the responsibility of the authors and do not necessarily represent the official views of the Patient- Centered Outcomes Research Institute. JAS was also supported by the resources and the use of facilities at the VA Medical Center at Birmingham, Alabama.

\section{Availability of data and materials}

Not applicable

\section{Authors' contributions}

JS and GAW designed the study; JS wrote the protocol; GAW, AH, and AK provided comments on the protocol. AH and AK performed the data analyses. All authors reviewed the analyses results and provided feedback related to the analyses. JS wrote the first draft of the paper. All authors edited and revised the manuscript and approved the final version for submission.

\section{Competing interests}

JAS has received research grants from Takeda and Savient and consultant fees from Savient, Takeda, Regeneron, Merz, Bioiberica, Crealta and Allergan pharmaceuticals, WebMD, UBM LLC, and the American College of Rheumatology. JAS serves as the principal investigator for an investigator-initiated study funded by Horizon pharmaceuticals through a grant to DINORA, Inc., a 501 (c)(3) entity. JAS is a member of the executive of OMERACT, an organization that develops outcome measures in rheumatology and receives arms-length funding from 36 companies; a member of the American College of Rheumatology's (ACR) Annual Meeting Planning Committee (AMPC); Chair of the ACR Meet-theProfessor, Workshop and Study Group Subcommittee; and a member of the Veterans Affairs Rheumatology Field Advisory Committee. GAW has received research grant and consultant fee from Bristol-Myers Squibb; consultant fees from Abbott, Amgen, UCB; Data Safety Monitoring for Novartis. Other authors $(\mathrm{AH}, \mathrm{AK})$ declare no financial conflict. None of the authors have any non-financial conflict.

All authors have disclosed potential conflicts of interest, have read the journal's policy on conflicts of interest and have read the journal's authorship agreement.

\section{Consent for publication \\ Not applicable.}

\section{Ethics approval and consent to participate}

The University of Alabama at Birmingham's Institutional Review Board approved this study and all investigations were conducted in conformity with ethical principles of research. The ethics committee waived the need for informed patient consent for this database study.

\section{Author details}

${ }^{1}$ Medicine Service, VA Medical Center, Birmingham, AL, USA. ${ }^{2}$ Department of Medicine at the School of Medicine, and Division of Epidemiology at the School of Public Health, University of Alabama at Birmingham, Faculty Office Tower 805B, 510 20th Street S, Birmingham, AL 35294, USA. ${ }^{3}$ Department of Orthopedic Surgery, Mayo Clinic College of Medicine, Rochester, MN, USA. ${ }^{4}$ Ottawa Heart Institute and the University of Ottawa, Ottawa, ON, Canada.

Received: 26 January 2016 Accepted: 26 August 2016 Published online: 13 September 2016

\section{References}

1. Feldman CH, Hiraki LT, Liu J, Fischer MA, Solomon DH, Alarcón GS, et al. Epidemiology and sociodemographics of systemic lupus erythematosus and lupus nephritis among US adults with Medicaid coverage, 2000-2004 Arthritis \& Rheumatism. 2013;65(3):753-63.

2. Odutola J, Ward MM. Ethnic and socioeconomic disparities in health among patients with rheumatic disease. Curr Opin Rheumatol. 2005;17(2):147-52.

3. Alarcon GS, Friedman AW, Straaton KV, Moulds JM, Lisse J, Bastian HM, et al. Systemic lupus erythematosus in three ethnic groups: III. A comparison of characteristics early in the natural history of the LUMINA cohort. LUpus in MInority populations: NAture vs. Nurture. Lupus. 1999;8(3):197-209. 
4. Centers for Disease Control and Prevention (CDC). Trends in deaths from systemic lupus erythematosus-United States, 1979-1998. MMWR Morb Mortal Wkly Rep. 2002;51(17):371-4.

5. Krishnan E, Hubert HB. Ethnicity and mortality from systemic lupus erythematosus in the US. Ann Rheum Dis. 2006;65(11):1500-5.

6. Institute of Medicine. 100 initial priority topics for comparative effectiveness research. http://www.nationalacademies.org/hmd/Reports/2009/ ComparativeEffectivenessResearchPriorities.aspx alone list of $100 \mathrm{cer}$ priorities - for web.pdf. National Academies Press; 2009.

7. Hahn BH, McMahon MA, Wilkinson A, Wallace WD, Daikh DI, Fitzgerald JD, et al. American College of Rheumatology guidelines for screening, treatment, and management of lupus nephritis. Arthritis Care Res. 2012;64(6):797-808.

8. Henderson L, Masson P, Craig JC, Flanc RS, Roberts MA, Strippoli GF, et al. Treatment for lupus nephritis. Cochrane Database Syst Rev. 2012;12:CD002922.

9. Helmick CG, Felson DT, Lawrence RC, Gabriel S, Hirsch R, Kwoh CK, et al. Estimates of the prevalence of arthritis and other rheumatic conditions in the United States. Part I. Arthritis Rheum. 2008;58(1):15-25.

10. Pogue J, Devereaux PJ, Thabane L, Yusuf S. Designing and analyzing clinical trials with composite outcomes: consideration of possible treatment differences between the individual outcomes. PLoS One. 2012;7(4):e34785.

11. Look ARG, Wing RR, Bolin P, Brancati FL, Bray GA, Clark JM, et al. Cardiovascular effects of intensive lifestyle intervention in type 2 diabetes. $\mathrm{N}$ Engl J Med. 2013;369(2):145-54

12. Ross S. Composite outcomes in randomized clinical trials: arguments for and against. Am J Obstet Gynecol. 2007;196(2):119 e1-6.

13. Agency for Healthcare Research and Quality. Methods guide for effectiveness and comparative effectiveness reviews. Rockville: Department of Health and Human Services; 2012. http://effectivehealthcare.ahrq.gov/ index.cfm/search-for-guides-reviews-and-reports/ ?pageaction=displayproduct\&productid $=318$.

14. Cassaday RD, Malik JT, Chang JE. Regression of Hodgkin lymphoma after discontinuation of a tumor necrosis factor inhibitor for Crohn's disease: a case report and review of the literature. Clin Lymphoma Myeloma Leuk. 2011;11(3):289-92

15. Singh JA, Noorbaloochi S, Tucker MD. Belimumab for systemic lupus erythematosus (protocol). Cochrane Database Syst Rev. 2013. DOl: 10.1002/14651858.CD010668

16. Hochberg MC. Updating the American College of Rheumatology revised criteria for the classification of systemic lupus erythematosus. Arthritis Rheum. 1997:40(9):1725.

17. Appel GB, Contreras G, Dooley MA, Ginzler EM, Isenberg D, Jayne D, et al. Mycophenolate mofetil versus cyclophosphamide for induction treatment of lupus nephritis. J Am Soc Nephrol. 2009;20(5):1103-12.

18. El-Shafey EM, Abdou SH, Shareef MM. Is mycophenolate mofetil superior to pulse intravenous cyclophosphamide for induction therapy of proliferative lupus nephritis in Egyptian patients? ClinExpNephrol. 2010;14(3):214-21.

19. Mitwalli AH, Al Wakeel JS, Hurraib S, Aisha A, Al SA, Alam A, et al. Comparison of high and low dose of cyclophosphamide in lupus nephritis patients: a long-term randomized controlled trial. Saudi J Kidney Dis Transpl. 2011;22(5):935-40.

20. Houssiau FA, D'Cruz D, Sangle S, Remy P, Vasconcelos C, Petrovic R, et al. Azathioprine versus mycophenolate mofetil for long-term immunosuppression in lupus nephritis: results from the MAINTAIN Nephritis Trial. Ann Rheum Dis. 2010;69(12):2083-9.

21. Higgins JP, Altman DG, Sterne JA. Chapter 8: assessing risk of bias in included studies. Cochrane Handbook for Systematic Reviews of Interventions Version 5.1.0 (updated March 2011). In: The Cochrane Collaboration. Higgins JP, Green S, editors. 2011. Available from http://handbook.cochrane.org/.

22. Lumley T. Network meta-analysis for indirect treatment comparisons. Stat Med. 2002;21(16):2313-24.

23. Psaty BM, Lumley T, Furberg CD, Schellenbaum G, Pahor M, Alderman $\mathbf{M H}$, et al. Health outcomes associated with various antihypertensive therapies used as first-line agents: a network meta-analysis. JAMA. 2003;289(19):2534-44.

24. Lu G, Ades AE. Combination of direct and indirect evidence in mixed treatment comparisons. Stat Med. 2004;23(20):3105-24.

25. Dias S, Sutton A, Welton N, Ades A, Golfinopoulos V, Kyrgiou M, et al. NICE DSU Technical Support Document 2: Generalised Linear Modelling Framework for Pairwise and Network Meta-Analysis of Randomised Controlled Trials. 2011.pp. 1-96. [cited 2012 June 13]. Available from: http://www.nicedsu.org.uk/TSD6\%20Software.final.08.05.12.pdf.
26. Dias S, Sutton A, Welton N, Ades A, Golfinopoulos V, Kyrgiou M, et al NICE DSU Technical Support Document 3: Heterogeneity: Subgroups, meta-regression, bias and bias-adjustment May 2011. pp. 1-24. [cited 2012 June 13]. Available from: http://www.nicedsu.org.uk/ TSD6\%20Software.final.08.05.12.pdf.

27. Cooper NJ, Sutton AJ, Morris D, Ades AE, Welton NJ. Addressing between-study heterogeneity and inconsistency in mixed treatment comparisons: Application to stroke prevention treatments in individuals with non-rheumatic atrial fibrillation. Stat Med. 2009;28(14):1861-81.

28. Turner RM, Thompson SG, Spiegelhalter DJ. Prior distributions for the intracluster correlation coefficient, based on multiple previous estimates, and their application in cluster randomized trials. Clin Trials. 2005;2(2):108-18.

29. Spiegelhalter D, Thomas A, Best N, Lunn D. WinBUGS User Manual. Version 1.4. 2003 [cited 2011 June 1]. Available from: http://www.mrc-bsu.cam.ac.uk/ wp-content/uploads/manual14.pdf.

30. Ades AE, Welton NJ, Caldwell D, Price M, Goubar A, Lu G. Multiparameter evidence synthesis in epidemiology and medical decision-making. J Health Serv Res Policy. 2008;13 Suppl 3:12-22.

31. Dias S, Sutton A, Welton N, Ades A, Golfinopoulos V, Kyrgiou M, et al. NICE DSU Technical Support Document 4: Inconsistency in Networks of Evidence Based on Randomised Controlled Trials. 2011. pp 1-39[cited 2012 June 13]. Available from: http://www.nicedsu.org.uk/TSD6\%20Software.final.08.05.12.pdf.

32. Puhan MA, Schunemann HJ, Murad MH, Li T, Brignardello-Petersen R, Singh JA, et al. A GRADE Working Group approach for rating the quality of treatment effect estimates from network meta-analysis. BMJ. 2014;349:95630

33. Radhakrishnan J, Moutzouris DA, Ginzler EM, Solomons N, Siempos, II, Appel GB. Mycophenolate mofetil and intravenous cyclophosphamide are similar as induction therapy for class V lupus nephritis. Kidney Int. 2010;77(2):152160. doi:10.1038/ki.2009.412

34. Mok CC, Ho CT, Siu YP, Chan KW, Kwan TH, Lau CS, Wong RW, Au TC. Treatment of diffuse proliferative lupus glomerulonephritis: a comparison of two cyclophosphamide-containing regimens. Am J Kidney Dis. 2001;38(2):256-64. doi:10.1053/ajkd.2001.26084

35. Hu W, Liu Z, Chen H, Tang Z, Wang Q, Shen K, Li L. Mycophenolate mofetil vs cyclophosphamide therapy for patients with diffuse proliferative lupus nephritis. Chin Med J. 2002;115(5):705-9.

36. Wang HY, Cui TG, Hou FF, Ni ZH, Chen XM, Lu FM, Xu FF, Yu XQ, Zhang FS, Zhao XZ, Zhao MH, Wang GB, Qian JQ, Cai GY, Zhu TY, Wang YH, Jiang ZP, Li YN, Mei CL, Zou WZ, China Leflunomide Lupus Nephritis Study G. Induction treatment of proliferative lupus nephritis with leflunomide combined with prednisone: a prospective multi-centre observational study. Lupus. 2008;17(7):638-44. doi:10.1177/0961203308089408.

37. Ginzler EM, Dooley MA, Aranow C, Kim MY, Buyon J, Merrill JT, et al. Mycophenolate mofetil or intravenous cyclophosphamide for lupus nephritis. N Engl J Med. 2005;353(21):2219-28.

\section{Submit your next manuscript to BioMed Central and we will help you at every step:}

- We accept pre-submission inquiries

- Our selector tool helps you to find the most relevant journal

- We provide round the clock customer support

- Convenient online submission

- Thorough peer review

- Inclusion in PubMed and all major indexing services

- Maximum visibility for your research

Submit your manuscript at www.biomedcentral.com/submit
) Biomed Central 